\begin{tabular}{|l|l|}
\hline Postprint Version & 1.0 \\
\hline Journal website & $\underline{\mathrm{http}: / / \text { journals.cambridge.org/action/displayAbstract?fromPage=online\&aid=79 }}$ \\
\hline Pubmed link & $\underline{24032}$ \\
\hline DOItp://Www.ncbi.nlm.nih.gov/pubmed/21073769 \\
\hline
\end{tabular}

This is a NIVEL certified Post Print, more info at http://www.nivel.eu

\title{
Social engagement and depressive symptoms of elderly residents with dementia: a cross-sectional study of 37 long- term care units
}

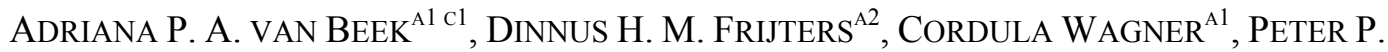 \\ GROENEWEGEN $^{A 1}$ AND MIEL W. RIBBE ${ }^{A 2}$ \\ ${ }^{a 1}$ NIVEL: Netherlands Institute for Health Services Research, Utrecht, The Netherlands \\ ${ }^{a 2}$ VU University/EMGO, Department of Nursing Home Medicine, Amsterdam, The Netherlands
}

\begin{abstract}
Background: Social engagement and depression are important outcomes for residents with dementia in long-term care. However, it is still largely unclear which differences in social engagement and depression exist in residents of various long-term care settings and how these differences may be explained. This study investigated the relationship between social engagement and depressive symptoms in long-term care dementia units, and studied whether differences in social engagement and depressive symptoms between units can be ascribed to the composition of the resident population or to differences in type of care setting.

Methods: Thirty-seven long-term care units for residents with dementia in nursing- and residential homes in the Netherlands participated in the study. Social engagement and depressive symptoms were measured for 502 residents with the Minimum Data Set of the Resident Assessment Instrument. Results were analyzed using multilevel analysis.

Results: Residents of psychogeriatric units in nursing homes experienced low social engagement. Depressive symptoms were most often found in residents of psychogeriatric units in residential homes. Multilevel analyses showed that social engagement and depressive symptoms correlated moderately on the level of the units. This correlation disappeared when the characteristics of residents were taken into account.

Conclusions: Social engagement and depressive symptoms are influenced not only by individual characteristics but also by the type of care setting in which residents live. However, in this study social engagement and depressive symptoms were not strongly related to each other, implying that separate interventions are needed to improve both outcomes.
\end{abstract}

\section{INTRODUCTION}

Social engagement and depression are important outcomes for residents in long-term care. Social engagement can be defined as social interactions with other residents and engagement in individual and social activities within the facility (Mor et al., 1995), and has been associated with a lower mortality risk among residents in nursing homes (Kiely and Flacker, 2003). Depression has also been linked to higher 
mortality rates in long-term care settings (Parmelee et al., 1992). Yet, studies show that residents in longterm care facilities frequently have low social engagement (Achterberg et al., 2003) and exhibit depressive symptoms which are often not recognized by nursing staff (Teresi et al., 2001).

Both social engagement and depressive symptoms are influenced by the residents' characteristics. For instance, cognitive problems are found to be predictive of both low social engagement (Bassuk et al., 1999) and depression (Gruber-Baldini et al., 2005; McCabe et al., 2006). Depression in elderly residents is associated with other characteristics such as age (Jongenelis, 2006), personality, social resources, and physical health (Reker, 1997; Eisses et al., 2004). Social engagement is also related to problems in physical (activities of daily living) functioning, although studies yield contrasting results (Drageset, 2004). The type of care setting to which residents are admitted seems to be another important element that influences social engagement (Schroll et al., 1997; Zimmerman et al., 2003; Dobbs et al., 2005) and depression (GruberBaldini et al., 2005; Jongenelis, 2006). For instance Jongenelis (2006) found that depressive symptoms were higher among residents in nursing homes in comparison to residential homes. This result was partly explained by the quality of care residents experienced. However, the nature of the differences in social engagement and depression that exist between various care settings remains unclear as does the extent to which these differences can be ascribed to variations in the composition of the resident population or to the type of care setting. This is especially true for residents with dementia even though, because of their illness, these residents are particularly susceptible to low social engagement and depressive symptoms.

In addition, little is known about the relationship between social engagement and depressive symptoms for residents with dementia. Studies have found that social engagement and depression are related to each other for residents in long-term care settings, with feelings of depression leading to low social engagement (Achterberg et al., 2003) or, conversely, depression leading to low social engagement (Tsai et al., 2009). Yet, these studies have focused on residents without (severe) cognitive problems. It remains unclear if this relationship is as strong for residents with dementia. For instance, apathy is a frequently observed behavior in dementia patients which influences both depression and daily activities (Yeager and Hyer, 2008).

In this study, we investigated social engagement and depressive symptoms for residents with dementia in different types of long-term care settings. First, we looked at the prevalence of low social engagement and depressive symptoms in units for residents with dementia in long-term care. Second, we studied whether differences in social engagement and depressive symptoms between units were the result of differences in the composition of the resident population or resulted from differences in the type of care setting, while taking into account a possible relationship between social engagement and depressive symptoms.

We addressed the following research questions:

- What is the prevalence of low social engagement and depressive symptoms in long-term care units for residents with dementia?

- 2. To what extent are differences in social engagement and depressive symptoms between units related to residents' characteristics and differences between care settings?

- 3. Is there a relationship between social engagement and depressive symptoms for residents with dementia in long-term care?

\section{METHODS}

\section{Setting}

Long-term care in the Netherlands is provided in nursing homes and residential homes. Nursing homes provide multi-disciplinary care for elderly residents with long-term, complex health problems (Ribbe, 1993). Residential homes in the Netherlands provide daily and basic medical care for infirm elderly (Eisses et al., 2004). However, the distinction between nursing homes and residential homes has become less clear over the years. Due to a growing number of elderly people in the Dutch population who require long-term care, complex multidisciplinary dementia care is provided in both nursing homes and residential homes in psychogeriatric units (Hoek et al., 2000). Residents in psychogeriatric units share living-rooms and often also their bedroom with other residents. Residential homes, in addition, provide dementia care in psychogeriatric group projects. These group projects also provide multidisciplinary care and aim to delay or prevent admission of residents to a nursing home. Residents who attend these group projects live in their own apartments in the facility, but spend most of their day in a small group setting of approximately 10 to 12 residents. 


\section{Participants}

In four regions of the Netherlands - West-Brabant, Rotterdam, Amsterdam and Stedendriehoek - longterm care facilities were asked to participate in the study on a voluntary basis. Data were collected between October 2002 and June 2003 from one dementia unit in each of 37 long-term care facilities. In total, 26 psychogeriatric units participated in the research: 16 units in nursing homes and 10 units in residential homes. In residential homes, 11 group projects also took part. The number of nursing staff on the units varied from 4 to 39 , with an average of 23 staff members. The group projects provided care to an average of 12 residents compared to 28 residents in psychogeriatric units in nursing homes.

According to Dutch regulations, no ethics approval for this study was necessary, as no intervention was investigated and data collection was based on proxy report and not collected directly from residents. We asked for written informed consent from legal representatives of all residents in the units prior to data collection. Only those residents for whom written informed consent was given were included in the study. In addition, client councils of the participating facilities were informed of the study in advance and anonymity and confidentiality for residents was guaranteed. Informed consent was given for 502 residents; this was $64 \%$ of the 792 residents who lived in the units at the time of data collection. Of these residents, 249 lived in nursing homes and 253 lived in residential homes. None of the residents was comatose at the time of data collection.

\section{Measures}

Data were collected over three days from each unit using the Resident Assessment Instrument (RAI) Minimum Data Set (MDS) for nursing home care (Morris et al., 1990). The MDS measures a wide array of characteristics of residents in nursing homes and several outcomes for residents and quality indicators can be computed (Morris et al., 1994). In this study, we used the Dutch version of the MDS 2.0 for residential long-term care. We used the following specific scales of the MDS:

\section{DEPRESSION RATING SCALE}

The MDS Depression Rating Scale (DRS) measures depressive symptoms of residents based on seven items: negative statements, persistent anger, expressions of what seem to be unrealistic fears, repetitive health complaints, repetitive anxious complaints/concerns, sad/pained/worried facial expressions, crying/tearfulness. The scores of the DRS indicate depression and can vary from 0 (no depressive symptoms) to 14 (frequent depressive symptoms). The internal consistency (Cronbach's $\alpha$ ) has been established at 0.71 and its sensitivity against a psychiatric diagnosis of depression was $91 \%$ (Burrows et al., 2000).

\section{INDEX OF SOCIAL ENGAGEMENT}

The Index of Social Engagement (ISE) (Mor et al., 1995) consists of six items that focus on the interaction with others, engagement in individual and group activities, goal-setting and involvement in the everyday life of the facility. The ISE adds the scores for all items (the score is 1 when the item is applicable) and has a range from 0 to 6 . A higher score stands for higher social engagement.

In addition, we measured age, gender, the presence or absence of a partner, and cognitive and physical functioning of residents with the MDS. Cognitive functioning was measured with the Cognitive Performance Scale (CPS) (Morris et al., 1994). The CPS consists of five items. Scores on the CPS range from 0 (intact) to 6 (very severe impairment). Problems in physical functioning were measured using the ADL (Activities of Daily Living) hierarchy index of the MDS (Morris et al., 1999; Gerritsen, 2004). The index ranges from "independent" (0) to "totally dependent on others" (6). The MDS data for each individual resident were collected by the researcher during an interview with a member of the nursing staff who knew the resident well and provided care to this resident. To measure possible problems in recognizing depressive symptoms, we looked for a diagnosis of depression and the use of antidepressants in the individual care plan of residents in relation to the scores on the DRS. Information on the type of care setting was collected at the start of data collection on each unit in an interview with the supervisor of the unit. During this interview information was collected on the number of residents and nursing staff on the units. 


\section{Analysis}

Differences in social engagement and depressive symptoms between units and individual residents were first analyzed using $\chi^{2}$ and t-tests. To study the relation between residents' characteristics, types of care setting, social engagement, and depressive symptoms, we then performed a multilevel analysis (Snijders and Bosker, 1999; Leyland and Groenewegen, 2003) using the statistical package MLwiN. To study the relationship between social engagement and depressive symptoms we analyzed a random intercept multiresponse model (Browne et al., 2007). This allowed us to look at social engagement and depressive symptoms separately while taking into account a possible relation with the outcomes.

The model consisted of three levels: units (level 3), individual residents on these units (level 2) and the relationship between social engagement and depressive symptoms within individual residents (level 1; see Technical Appendix for a description of the model). We analyzed the model in two steps: (i) after the empty model, we studied residents' characteristics (model 1); and (ii) we entered type of care setting into the analyses (model 2).

\section{RESULTS}

\section{Characteristics of residents}

Analysis showed that the 37 units participating in the study did not differ from nursing homes and residential homes elsewhere in the Netherlands in the age and qualifications of nursing staff, and the number of shared bedrooms (Mathijssen et al., 2004). Furthermore, residents in our sample did not differ from the psychogeriatric resident population in nursing homes in the Netherlands with regard to their psychosocial and somatic care requirements (Mathijssen et al., 2004).

Residents of the units differed in several important ways (Table 1). Residents of psychogeriatric units in nursing homes were younger in comparison with residents of units in residential homes. The oldest residents were found in the group projects. These units also had the highest percentage of women and fewer residents with a partner compared to residents of psychogeriatric units in nursing homes and residential homes. There were also differences in cognitive functioning and physical functioning between residents of the units. Although psychogeriatric units in nursing homes and residential homes are designed for the same type of residents, residents of psychogeriatric units in nursing homes in particular suffered from severe problems in cognitive functioning (scores 5 and 6). Mild to moderate cognitive problems were most frequently reported in residents of residential homes. A majority of residents of psychogeriatric units in nursing homes were dependent on others for their physical functioning. Of the residents who participated in group projects in residential homes, the majority were independent in terms of their physical functioning.

\section{[TABLE 1]}

\section{Prevalence of social engagement and depressive symptoms}

Table 2 shows the results for social engagement and depressive symptoms of residents. Residents with dementia in psychogeriatric units in nursing homes had less social engagement compared to residents in residential homes. Scores on the ISE can be dichotomized in two categories: low social engagement (scores 0-2) and moderate to high social engagement (scores 3-6) (Achterberg et al., 2003). When we used this dichotomization, $42.6 \%$ of residents of psychogeriatric units in nursing homes had low social engagement. In residential homes the percentage of residents with low social engagement was significantly lower, with $27.1 \%$ of residents in psychogeriatric units and $23.0 \%$ of residents in group projects.

\section{[TABLE 2]}

Scores on depressive symptoms were higher for residents of psychogeriatric units in residential homes, compared to residents of psychogeriatric units in nursing homes and residents in group projects. Burrows et al. (2000) demonstrated that scale scores of 3 or more on the DRS indicate minor and major depressive disorders. In psychogeriatric units in nursing homes, $41.8 \%$ of residents had a score of 3 or higher. In residential homes, $57.1 \%$ of the residents of psychogeriatric units and $46.9 \%$ of residents in group projects scored 3 or higher on this scale. Of these residents, only a few were diagnosed with depression according to their individual care plan (varying between 5\% of residents of psychogeriatric units in nursing homes and 
residential homes, and $0 \%$ in the group projects; data not shown in Table 2). Slightly more residents with a DRS score of 3 or higher used antidepressants: $14 \%-16 \%$ of residents of psychogeriatric units in nursing homes and residential homes, and $8 \%$ of residents in group projects (not shown in Table 2).

Next, we studied whether differences in social engagement and depressive symptoms between units were related to differences in the composition of the resident population on the units or to differences in care setting. To investigate a possible relation between social engagement and depressive symptoms, we analyzed a multilevel, multi-response model with social engagement and depressive symptoms as the dependent variables (Table 3). As expected, social engagement and depressive symptoms varied significantly between individual residents. However, there were also significant differences between the units (see variance components for the empty model in Table 3). Social engagement and depressive symptoms were positively related to each other on the level of the units (correlation multilevel analyses 0.34), indicating that units with higher scores on social engagement also had higher scores on depressive symptoms. On the level of the residents, the correlation between both outcomes was negative but not very strong (correlation multilevel analyses -0.10); residents with high social engagement had less depressive symptoms. Of the variance, $21 \%$ and $16 \%$ could be ascribed to differences on the level of the units (as presented by the intra-class correlation in the empty model in Table 3).

\section{[TABLE 3]}

\section{Factors that influence social engagement and depressive symptoms}

We first entered residents' characteristics into the model: age, sex, having a partner, physical functioning and cognitive functioning (Model 1, Table 3). As expected, social engagement of residents was related to physical and cognitive functioning. Residents who were more dependent on others in their physical functioning and residents who experienced more cognitive problems had less social engagement $(\mathrm{p}<0.05)$. Physical and cognitive functioning were not significantly related to depressive symptoms. We did find a significant relationship between depressive symptoms and having a partner. Residents with a partner experienced more depressive symptoms $(\mathrm{p}<0.05)$. Gender and age were not significantly related, either to social engagement or depressive symptoms. When we added residents' characteristics to the model, differences remained significant both on the level of units and on the level of individual residents (see variance components Model 1, Table 3). When taking residents' characteristics into account, the correlation between social engagement and depressive symptoms was low both on the level of units and on the level of individual residents (correlation multilevel analyses were 0.11 and -0.11 , respectively).

Second, we entered the type of care setting into the model to see if social engagement and depression differed between care settings while taking residents' characteristics into account (Model 2, Table 3). Variations in types of care setting were related to depressive symptoms. Residents of psychogeriatric units in residential homes had a higher score on the Depression Rating Scale in comparison with residents of psychogeriatric units in nursing homes, even when we adjusted for residents' characteristics $(\mathrm{p}<0.05)$. By adding type of care setting into the model for depressive symptoms, the variance between units decreased and was no longer significant. No such effect was found for social engagement, the variance on unit-level for this outcome remained significant (see variance components Model 2, Table 3). By adding residents' characteristics and type of care setting the intra-class correlation for units was reduced to $11 \%$ for social engagement and $6 \%$ for depressive symptoms. When taking the type of care setting into account, the correlation between social engagement and depressive symptoms remained low on the level of units and on the level of individual residents (correlation multilevel analyses were -0.11 for both levels).

\section{DISCUSSION}

Differences between types of care setting were found for both social engagement and depressive symptoms. Low social engagement was most often found in residents of psychogeriatric units in nursing homes. Depressive symptoms were most often found in residents of psychogeriatric units in residential homes. Differences between units in social engagement and depressive symptoms were mainly due to differences in the composition of the resident population of the units. However, residents of psychogeriatric units in residential homes scored higher on depressive symptoms compared to residents in other settings even after we controlled for residents' characteristics. We also studied the relationship between depressive 
symptoms and social engagement of residents with dementia in long-term care. To this end, we analyzed both outcomes in a multilevel multi-response model. Although we found a positive correlation between both outcomes in our initial model, this correlation ceased to exist when we took residents' characteristics into account.

As expected, residents with severe problems in cognitive and physical functioning had less social engagement. Unexpectedly, in our study depressive symptoms were only significantly related to having a partner and not to other residents' characteristics. Residents with a partner had higher scores on depressive symptoms in comparison to those without a partner. This may be due to several factors. In the Netherlands, residents who are admitted to a psychogeriatric unit live separated from their partners. This means that in our sample, only the 20 residents with a partner in group projects lived together with their partner. The other 124 residents with a partner lived separately, which may have led to depressive symptoms. However, an explanation can also be found in the initial reason for admission to the psychogeratric unit. Residents with dementia living at home with a spouse or partner are more often institutionalized for behavior-related problems, such as depressive symptoms, as changes in behavior of the person with dementia lead to increased caregiver burden (Gaugler et al., 2000).

The percentage of residents with scores of 3 or higher on the DRS is comparable to the $44 \%$ of residents with depressive symptoms found in an earlier Dutch study (Jongenelis, 2006). Contrary to our results, Jongenelis found more depressive symptoms among residents in nursing homes than in residential homes. This may be explained by the fact that Jongenelis excluded residents with moderate to severe dementia, while those residents were the focus of the present study. Another explanation may be found in our use of the DRS to measure depressive symptoms and the adequacy with which depressive symptoms were detected and recognized by nursing staff. There are concerns about the reliability of the DRS for residents with cognitive impairment, although internal consistency seems to be sufficient (Gerritsen, 2004). On the other hand, it is suggested that the DRS may be particularly appropriate as it was developed specifically for the frail nursing home population and is less dependent on physical conditions (Verkaik et al., 2010). Furthermore, nursing staff in the Netherlands are not often trained to identify symptoms of depression. To study the adequacy of the detection of depressive symptoms in our sample with the DRS, we looked at the internal consistency of the DRS items in the three settings. Although the internal consistency was sufficient for all three settings, we found that internal consistency was highest for psychogeriatric units (Cronbach's $\alpha$ $=0.70$ ) and group projects (Cronbach's $\alpha=0.69$ ) in residential homes. In psychogeriatric units in nursing homes, internal consistency on the DRS was 0.62 suggesting that differences between the care settings may indeed be caused by differences in the adequacy of detection by nursing staff. The lack of training in identifying depressive symptoms may also account for the fact that few of the residents in our study were diagnosed with depression or treated with antidepressants. This under-diagnosis of depressive symptoms for elderly residents with dementia is in line with other studies (Teresi et al., 2001).

This under-diagnosis of depressive symptoms might also partly explain the lack of a relationship between depressive symptoms and social engagement in our sample. The addition of another depression measure to the study would have been beneficial to the interpretation of our results. Unfortunately, this was not possible in the current study design.

This study has two other important limitations. Because we used a cross-sectional design, it was not possible to measure changes in residents throughout their stay in the unit and a causal relationship between social engagement and depressive symptoms, resident characteristics and type of care setting could not be tested. In addition, due to the cognitive problems of the residents in our study, it was impossible to measure social engagement and depressive symptoms through self-report. Instead we used measurements based on proxy report: social engagement and depressive symptoms were assessed by nursing staff. We have no information on how nursing staff reports relate to residents' perception of their social engagement and depressive symptoms.

In summary, our results show that depressive symptoms and low social engagement are important problems in various long-term care settings for residents with dementia. Depressive symptoms and social engagement are not only influenced by the residents' characteristics but also by the setting in which residents live. Even when we control for differences in residents' characteristics, differences between units remain. The results indicate that both outcomes may be improved by focusing on care-processes at unit level as well as on the care for individual residents. In addition, by analyzing social engagement and depressive symptoms in a multilevel multi-response model we take a first but important step in 
disentangling both outcomes for residents with dementia. In this study, social engagement and depressive symptoms are not strongly related to each other, implying that separate interventions are needed to improve both outcomes for residents with dementia. Further research is required to determine which specific aspects of psychogeriatric units influence social engagement and depressive symptoms, while taking differences in the population of residents into account.

\section{TECHNICAL APPENDiX: MULTILEVEL MULTI-RESPONSE MODEL}

To answer our research questions a three-level multi-response model for the two continuous responses was fitted. This allowed us to analyze the two responses conditional on covariates and on each other. The model was constructed as follows:

$$
\begin{gathered}
y_{1 i j}=\left(X_{1} \beta_{1}\right)_{i j}+\left(Z_{1} \beta_{1}\right)_{j}+\mu_{1 j}+e_{1 i j} \\
y_{2 i j}=\left(X_{2} \beta_{2}\right)_{i j}+\left(Z_{2} \beta_{2}\right)_{j}+\mu_{2 j}+e_{2 i j} \\
\left(\begin{array}{l}
e_{1 i j} \\
e_{2 i j}
\end{array}\right) \sim N\left(0, \Omega_{e}\right), \Omega_{e}=\left(\begin{array}{c}
\sigma_{e 1}^{2} \\
\sigma_{e 12} \sigma_{e 2}^{2}
\end{array}\right) \\
\left(\begin{array}{l}
\mu_{1 j} \\
\mu_{2 j}
\end{array}\right) \sim N\left(0, \Omega_{\mu}\right), \Omega_{\mu}=\left(\begin{array}{c}
\sigma_{\mu 1}^{2} \\
\sigma_{\mu 12} \sigma_{\mu 2}^{2}
\end{array}\right)
\end{gathered}
$$

where

- $\quad \mathrm{i}=$ residents $(1 \ldots \mathrm{n})$

- $\mathrm{j}=\operatorname{units}(1 \ldots \mathrm{N})$

- $\mathrm{X}=$ covariates measured at the level of residents

- $\mathrm{Z}=$ covariates measured at the unit level

- $\mathrm{e}=$ residents residual

- $\sigma^{2} \mathrm{e}=$ between residents within unit variance

- $\sigma \mathrm{e}_{12}=$ covariance between residents residuals for the two dependents responses

- $\mu=$ unit residual

- $\sigma_{\mu}^{2}=$ between unit variance

- $\sigma_{\mu 12}=$ covariance between unit residuals for the two dependents responses

- $\quad$ subscript 1 = indicator first dependent response

- $\quad$ subscript 2 = indicator second dependent response

Level 1 stays unspecified; no variance was defined at this level. If in the above model the two covariances are equal to zero, the results would be the same as if the dependent variables were analyzed separately. The correlation between the two dependent variables at the residents' level was calculated as: $\frac{\sigma_{s, 12}}{\sqrt{\sigma_{e 1}^{2} * \sigma_{2 e}^{2}}}$. The ICC was calculated as: $\frac{\sigma_{u}^{2}}{\sigma_{c}^{2}+\sigma_{u t}^{2}}$, so the ICCs for the two dependent variables are corrected for the dependence (covariance) at the residents' level.

\section{CONFLICT OF INTEREST}

None.

\section{DESCRIPTION OF AUTHORS' ROLES}

A. van Beek planned the study, collected, analyzed and interpreted the data and wrote the paper. C. Wagner, D. Frijters, M. Ribbe and P. Groenewegen helped with the study design, including instrumentation, data analysis and revision of the paper. 


\section{ACKNOWLEDGMENTS}

The study was supported by a grant from the Netherlands Organization for Scientific Research and the Netherlands Organization for Health Research and Development. The authors would like to thank Peter Spreeuwenberg of the Netherlands Institute of Health Services Research for his help with the multilevel analyses.

\section{REFERENCES}

Achterberg, W., Pot, A. M., Kerkstra, A., Ooms, M., Muller, M. and Ribbe, M. W. (2003). The effect of depression on social engagement in newly admitted Dutch nursing home residents. The Gerontologist, 43, 213-218.

Bassuk, S. S., Glass, T. A. and Berkman, L. F. (1999). Social disengagement and incident cognitive decline in community-dwelling elderly persons. Annals of Internal Medicine, 131, 165-173.

Browne, W. J., McCleerly, R. H., Sheldon, B. C. and Pettifor, R. A. (2007). Using cross-classified multivariate mixed response models with application to life history traits in great tits (Parus major). Statistical Modelling, 7, 1-23.

Burrows, A. B., Morris, J. N., Simon, S. E., Hirdes, J. P. and Phillips, C. (2000). Development of Minimum Data Set-based depression rating scale for use in nursing homes. Age and Ageing, 29, 165-172.

Dobbs, D. et al. (2005). Characteristics associated with lower activity involvement in long-term care residents with dementia. The Gerontologist, 45 (S1), 81-86.

Drageset, J. (2004). The importance of activities of daily living and social contact for loneliness; a survey among residents in nursing homes. Scandinavian Journal of Caring Sciences, 18, 65-71.

Eisses, A. M. H., Kluiter, H., Jongenelis, K., Pot, A. M., Beekman, A. T. F. and Ormel, J. (2004). Risk indicators of depression in residential homes. International Journal of Geriatric Psychiatry, 19, 634-640.

Gaugler, J. E. et al. (2000). Predictors of institutionalization of cognitively impaired elders: family help and the timing of placement. Journal of Gerontology: Psychological Sciences, 55B, P247-P255.

Gerritsen, D. L. (2004). Quality of life and its measurement in nursing homes. Unpublished PhD. thesis, Amsterdam: VU.

Gruber-Baldini, A. L., Zimmerman, S., Boustani, M., Watson, L. C., Williams, C. S. and Reed, P. S. (2005). Characteristics associated with depression in long-term care residents with dementia. The Gerontologist, 45 (S1), 50-55.

Hoek, J. F., Penninx, B. W. J. H., Ligthart, G. J. and Ribbe, M. W. (2000). Health care for older persons, a country profile: The Netherlands. Journal of the American Geriatrics Society, 48, 214-217.

Jongenelis, K. (2006). Depression in Dutch nursing homes: The Amsterdam Groningen Elderly Depression Study. Unpublished PhD thesis, Amsterdam: VU.

Kiely, D. K. and Flacker, J. M. (2003). The protective effect of social engagement on 1-year mortality in a long-stay nursing home population. Journal of Clinical Epidemiology, 56, 472-478.

Leyland, A. H. and Groenewegen, P. P. (2003). Multilevel modelling and public health policy. Scandinavian Journal of Public Health, 31, 267-274.

Mathijssen, S. W., Kwartel, A. J. J. Van Der and Pepels, C. G. M. (2004). Brancherapport Care 2000-2003. Den Haag: Ministerie van Volksgezondheid, Welzijn en Sport.

McCabe, M. P., Davison, T., Mellor, D., George, K., Moore, K. and Ski, C. (2006). Depression among older people with cognitive impairment: prevalence and detection. International Journal of Geriatric Psychiatry, 21, 633-644.

Mor, V. et al. (1995). The structure of social engagement among nursing home residents. Journal of Gerontology: Psychological Sciences, 50B, 1-8.

Morris, J. N., Hawes, C. and Fries, B. E. (1990). Designing the national Resident Assessment Instrument for nursing facilities. The Gerontologist, 30, 293-307.

Morris, J. N. et al. (1994). MDS Cognitive Performance Scale. Journal of Gerontology: Medical Sciences, 49, M174-M182.

Morris, J. N., Fries, B. E. and Morris, S. A. (1999). Scaling ADL's within the MDS. Journal of Gerontology: Medical Sciences, 54, M546-M553.

Parmelee, P. A., Katz, I. R. and Lawton, M. P. (1992). Depression and mortality among institutionalized aged. Journal of Gerontology, 47, 3-10.

Reker, G. T. (1997). Personal meaning, optimism and choice: existential predictors of depression in community and institutional elderly. The Gerontologist, 37, 709-716.

Ribbe, M. W. (1993). Care for the elderly: the role of the nursing home in the Dutch health care system. International Psychogeriatrics, 5, 213-222.

[CJO Abstract] 
Beek, A.P.A. van, Frijters, D.H.M., Wagner, C., Groenewegen, P.P., Ribbe, M.W. Social engagement and depressive symptoms of elderly residents with dementia: a cross-sectional study of 37 long-term care units International Psychogeriatrics: 2011, 23(4), 625-633

Schroll, M., Jónsson, P., Mor, V., Berg, K. and Sherwood, S. (1997). An international study of social engagement among nursing home residents. Age and Ageing, 26 (S2), 55-59.

Snijders, T. A. B. and Bosker, R. J. (1999). Multilevel Analysis: An Introduction to Basic and Advanced Multilevel Modelling. London: Sage Publications.

Teresi, J., Abrams, R., Holmes, D., Ramirez, M. and Eimicke, J. (2001). Prevalence of depression and depression recognition in nursing homes. Social Psychiatry and Psychiatry Epidemiology, 36, 613-620.

Tsai, C. et al. (2009). Depression is the strongest independent risk factor for poor social engagement among Chinese elderly veteran assisted-living residents. Journal of the Chinese Medical Association, 72, 478-483.

Verkaik, R., Francke, A. L., Meijel, B. van, Spreeuwenberg, P. M. M., Ribbe, M. W. and Bensing, J. M. (2010). The effects of a nursing guideline on depression in psychogeriatric nursing home residents with dementia. International Journal of Geriatric Psychiatry. In press.

Yeager, C. A. and Hyer, L. (2008). Apathy in dementia: relations with depression, functional competence, and quality of life. Psychological Reports, 102, 718-722.

Zimmerman, S. et al. (2003). Social engagement and its relationship to service provision in residential care and assisted living. Social Work Research, 27, 6-18. 
Table 1. Characteristics of residents with dementia in different care settings $(N=502)$

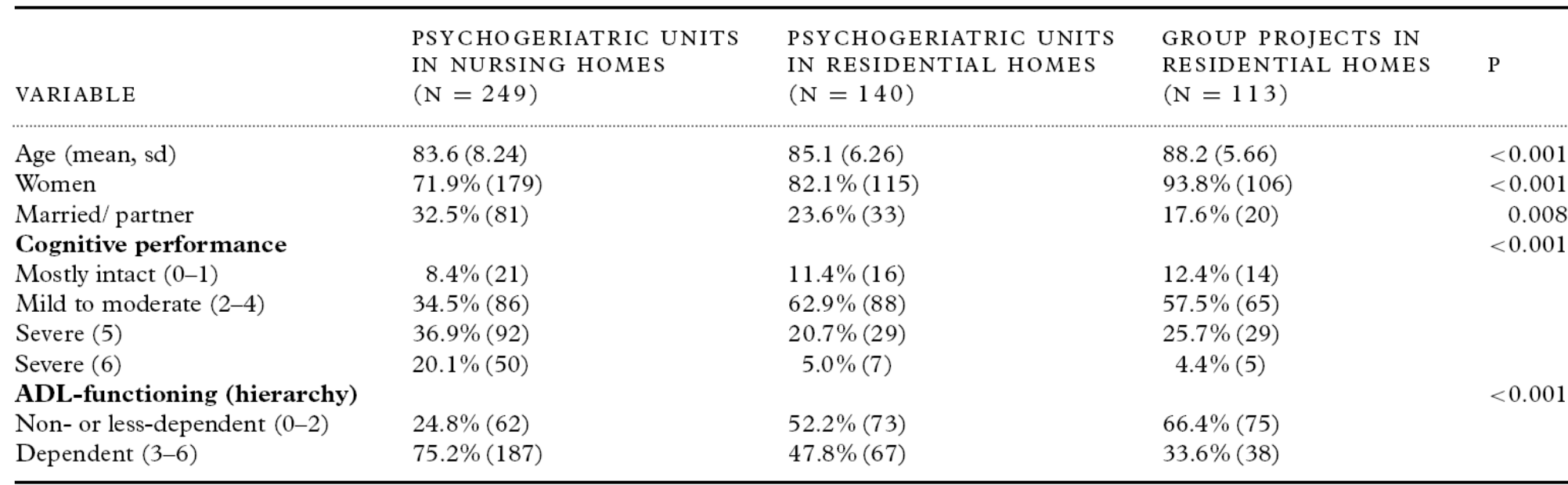

$\mathrm{ADL}=$ activities of daily living

Table 2. Social engagement and depressive symptoms of residents with dementia in different care settings $(\mathrm{N}=502)$

\begin{tabular}{llcc}
\hline & $\begin{array}{l}\text { PSYCHOGERIATRIC UNITS } \\
\text { IN NURSING HOMES } \\
\text { VARIABLE }\end{array}$ & $\begin{array}{l}\text { PSYCHOGERIATRIC UNITS } \\
\text { IN RESIDENTIAL HOMES } \\
(\mathrm{N}=140)\end{array}$ & $\begin{array}{l}\text { GROUP PROJECTS IN } \\
\text { RESIDENTIAL HOMES } \\
(\mathrm{N}=113)\end{array}$ \\
Social engagement (mean, sd) & $2.6(1.67)$ & $3.5(1.75)$ & $3.5(1.54)$ \\
Low social engagement (0-2) & $42.6 \%(106)$ & $27.1 \%(38)$ & $23.0 \%(26)$ \\
Moderate to high social engagement (3-6) & $57.4 \%(143)$ & $72.9 \%(102)$ & $77.0 \%(87)$ \\
Depressive symptoms (mean, sd) & $2.6(2.25)$ & $3.6(2.79)$ & $2.8(2.64)$ \\
No depressive symptoms (0-2) & $58.2 \%(145)$ & $42.9(60)$ & $53.1 \%(60)$ \\
Depressive symptoms $(\geq 3)$ & $41.8 \%(104)$ & $57.1(80)$ & $46.9 \%(53)$ \\
\hline
\end{tabular}


Beek, A.P.A. van, Frijters, D.H.M., Wagner, C., Groenewegen, P.P., Ribbe, M.W. Social engagement and depressive symptoms of elderly residents with dementia: a cross-sectional study of 37 long-term care units International Psychogeriatrics: 2011, 23(4), 625-633

Table 3. results of the multi-level multi-response analyses for social engagement ${ }^{*}$ and depressive symptoms ${ }^{* *}$ of residents $(\mathrm{N}=502)$ of the units $(\mathrm{N}=37)$

\begin{tabular}{|c|c|c|c|c|c|c|}
\hline & \multicolumn{2}{|c|}{ EMPTY MODEL } & \multicolumn{2}{|c|}{ MODEL 1} & \multicolumn{2}{|c|}{ MODEL 2} \\
\hline & SOCIAL & DEPRESSIVE & SOCIAL & DEPRESSIVE & SOCIAL & DEPRESSIVE \\
\hline & $\begin{array}{l}\text { ENGAGEMENT } \\
(0-6)\end{array}$ & $\begin{array}{l}\text { SYMPTOMS } \\
(0-14)\end{array}$ & $\begin{array}{l}\text { ENGAGEMENT } \\
(0-6)\end{array}$ & $\begin{array}{l}\text { SYMPTOMS } \\
(0-14)\end{array}$ & $\begin{array}{l}\text { ENGAGEMENT } \\
(0-6)\end{array}$ & $\begin{array}{l}\text { SYMPTOMS } \\
(0-14)\end{array}$ \\
\hline & $\mathrm{B}(\mathrm{SE})$ & $\mathrm{B}(\mathrm{SE})$ & $\mathrm{B}(\mathrm{SE})$ & $\mathrm{B}(\mathrm{SE})$ & B (SE) & $\mathrm{B}(\mathrm{SE})$ \\
\hline Intercept & $3.114(0.125)$ & $2.937(0.173)$ & $3.525(0.834)$ & $3.675(1.390)$ & $3.487(0.836)$ & $3.563(1.385)$ \\
\hline \multicolumn{7}{|l|}{ Characteristics of residents } \\
\hline Age & & & $0.017(0.010)$ & $-0.010(0.016)$ & $0.016(0.010)$ & $-0.013(0.016)$ \\
\hline Female (ref: male) & & & $0.022(0.172)$ & $0.283(0.287)$ & $-0.001(0.173)$ & $0.223(0.288)$ \\
\hline Partner (ref: no partner) & & & $-0.167(0.163)$ & $0.807(0.272)$ & $-0.167(0.163)$ & $0.807(0.270)$ \\
\hline Problems in ADL & & & $-0.170(0.043)$ & $-0.048(0.071)$ & $-0.164(0.044)$ & $-0.039(0.073)$ \\
\hline Problems in cognitive performance & & & $-0.369(0.053)$ & $-0.060(0.089)$ & $-0.364(0.053)$ & $-0.046(0.088)$ \\
\hline \multicolumn{7}{|l|}{ Care setting } \\
\hline Psychogeriatric unit in residential home (ref: & psychogeriatric ur & nit in nursing hom & & & $0.370(0.242)$ & $1.016(0.365)$ \\
\hline Group setting in residential home (ref: psych & ogeriatric unit in 1 & nursing home) & & & $0.180(0.254)$ & $0.272(0.387)$ \\
\hline \multicolumn{7}{|c|}{ Variance components } \\
\hline Units & $0.383(0.135)$ & $0.665(0.258)$ & $0.226(0.089)$ & $0.593(0.239)$ & $0.200(0.083)$ & $0.368(0.187)$ \\
\hline Residents & $2.556(0.168)$ & $5.788(0.379)$ & $2.038(0.134)$ & $5.683(0.372)$ & $2.040(0.134)$ & $5.709(0.374)$ \\
\hline Correlation between outcomes for units & \multicolumn{2}{|c|}{0.34} & \multicolumn{2}{|c|}{0.11} & \multicolumn{2}{|c|}{-0.11} \\
\hline Correlation between outcomes for residents & \multicolumn{2}{|c|}{-0.10} & \multicolumn{2}{|c|}{-0.11} & \multicolumn{2}{|c|}{-0.11} \\
\hline ICC for units & $21 \%$ & $16 \%$ & $10 \%$ & $11 \%$ & $11 \%$ & $6 \%$ \\
\hline
\end{tabular}

"A higher score represents more social engagement; ${ }^{\star \star} \mathrm{A}$ higher score represents more depressive symptoms. $\mathrm{ADL}=$ activities of daily living; $\mathrm{ICC}=$ intra-class correlation 